\title{
Circular RNA circANKS1B as the Sponge of miR- 152-3p Promotes Prostate Cancer Progression by up-Regulating TGF-a Expression
}

\section{Liangjun Tao}

First Affiliated Hospital of Anhui Medical University

\section{Xinyuan Pan}

The Second People Hospital of Wuhu

Jiawei Wang

The Second people Hospital of Wuhu

\section{Li Zhang}

First Affiliated Hospital of Anhui Medical University

\section{Lingsong Tao}

The Second People Hospital of Wuhu

Chaozhao Liang ( $\square$ liang_chaozhao@ahmu.edu.cn )

Anhui Medical University https://orcid.org/0000-0003-2317-1323

Primary research

Keywords: circANKS1B, cell invasion, miR-152-3p, TGF-a, prostate cancer

Posted Date: August 28th, 2020

DOI: https://doi.org/10.21203/rs.3.rs-66869/v1

License: (1) This work is licensed under a Creative Commons Attribution 4.0 International License. Read Full License 


\section{Abstract}

Background: Growing studies indicate that circRNAs play critical roles in human diseases, and show great potential as biomarkers and therapeutic targets. This study aims to investigate the expression and function of circANKS1B in prostate cancer (PC).

Methods: The expression of circANKS1B and miRNA-152-3p were determined by real-time qRT-PCR. The cell migration and invasion were measured by transwell assay. The interaction between circANKS1B and miR-152-3p was confirmed by dual-luciferase reporter gene assay. Rescue experiments were conducted to demonstrate whether circANKS1B regulated the migration and invasion of PC cells by the circANKS1BmiR-152-3p-TGF-a pathway.

Results: The expression of circANKS1B was dramatically up-regulated both in PC cells and tissues. Moreover, high circANKS1B expression was associated with a poor prognosis of PC patients. Dualluciferase reporter assay indicated that circABKS1B directly bound to miRNA-152-3p. Furthermore, circANKS1B negatively regulated miR-152-3p expression. Knockdown of circANKS1B remarkably suppressed PC cells invasion and TGF-a expression, while the effects of circANKS1B silencing were reversed by miR-152-3p deficiency. In addition, the impact of miR-152-3p silencing on PC cell invasion was also abrogated by TGF-a deficiency. In all, circANKS1B as the sponge of miR-152-3p promotes prostate cancer progression by up-regulating TGF-a expression.

Conclusion: Our findings reveal that circANKS1B could be a potential prognostic biomarker and therapeutic target of PC.

\section{Background}

Prostate cancer (PC) is the second most frequent cancer and the fifth leading cause of cancer death in men worldwide, with an estimated 1.3 million new cases and 359,000 deaths in 2018 [1]. However, the incidence and mortality of prostate cancer are still on the rise. In 2019, 174,650 new cases and 31,620 deaths of PC were reported in the United States [2]. Radical prostatectomy or radical radiotherapy is the main treatment for localized PC, and a comprehensive therapy based on androgen deprivation therapy (ADT) is the treatment for advanced disease [3]. Once hormone resistance occurs, advanced PC often develops into metastatic PC. Disease relapse and metastasis, as well as development of hormone refractory disease, remain the major causes of death in PC patients. Therefore, it is imperative to investigate the molecular mechanisms involved in PC progression and find novel therapeutic modalities as well as prognostic biomarkers.

Circular RNAs (circRNAs), a kind of endogenous noncoding RNA characterized by covalently closed loop structures without 5'-cup structure and $3^{\square}$-polyadenylated tail, are widely found in mammals and highly conserved as well as stable [4]. CircRNAs play critical roles in human physiological and pathological conditions, the known functions include sequestration of microRNAs or proteins, modulation of transcription and interference with splicing, as well as even translation to produce polypeptides [5-8]. 
Growing evidence showed that circRNAs were involved in various cancer biological processes, including proliferation, differentiation, apoptosis, and metastasis [9, 10]. Moreover, because of their conservation, abundance and tissue specificity, circRNAs also show great potential as diagnostic biomarkers in cancers [11]. CircANKS1B (hsa_circ_0007294), a circRNA originated from exons 5 to 8 of the ANKS1B gene, is significantly overexpressed and promotes the metastasis of breast cancer [12]. Moreover, Li et al. [13] reported circANKS1B was increased and enforced cell migration and invasion in colorectal cancer. However, the specific expression and role of circANKS1B in PC has not been explored.

Our study attempted firstly to investigate the expression and function of circANKS1B in PC. We observed that circANKS1B was dramatically overexpressed both in PC cells and tissues. Moreover, high circANKS1B expression was associated with a poor prognosis of PC patients. Functional analysis showed that circANKS1B as the sponge of miR-152-3p could facilitate cell migration and invasion by upregulating TGF-a expression in PC. Our findings reveal that circANKS1B could be a potential prognostic biomarker and therapeutic target of PC.

\section{Material And Methods}

\section{Clinical ethics and human tissues}

Forty-two pairs PC tissues and adjacent non-tumor tissues were collected from patients who underwent radical prostatectomy at the Department of Urology, Second people hospital of Wuhu (Wuhu, China). All of the samples were collected after surgery, immediately placed in liquid nitrogen, and stored at $-80^{\circ} \mathrm{C}$ before further analysis. All PC tissues were confirmed by pathological study post operatively. Adjacent non-tumor tissue was also resected simultaneously, and half of it was sent to pathological inspection to rule out contamination of tumor. Patient follow-up was performed in the outpatient department by phone. Informed consent was obtained from each patient and the study was approved by the ethics committee

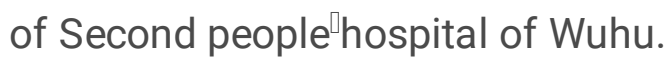

\section{Cell lines and cell culture}

Human PC cell lines (C4-2, LNCaP, DU145 and PC-3) and human prostate epithelial cell line (RWPE-1) were obtained from the Chinese Academy of Sciences Cell Bank (Shanghai, China). PC cell lines were maintained in RPMI-1640 medium. RWPE-1 cells were maintained in DMEM. The both media contain $10 \%$ FBS, $100 \mathrm{U} / \mathrm{mL}$ penicillin, and $0.1 \mathrm{mg} / \mathrm{mL}$ streptavidin. All cells were incubated at $37^{\circ} \mathrm{C}$ in a humidified atmosphere containing $5 \% \mathrm{CO}_{2}$.

\section{Cell transfection}

MiR-152-3p mimics, miR-152-3p inhibitor, si-TGF- $a$ and negative control (NC) were purchased from GenePharma Biotechnology (Shanghai, China). siRNA (small interfering-RNA) targeting circANKS1B (5囚GAAGCCAGAGTGTAACAGA-3区) and siRNA NC were synthesized by Invitrogen. Transfection of cells was 
performed with Lipofectamine 2000 Reagent (Invitrogen) according to the manufacturer's protocol. Briefly, $1 \times 10^{6}$ cells were seeded in six-well plates at $70 \%$ confluence a day before transfection.

\section{RNA isolation and real-time qRT-PCR}

Total RNA was isolated from PC tissues and cells using Trizol (Invitrogen) according to the manufacturer's instructions for circRNA and miRNA analyses. MiR-152a-3p real-time qRT-PCR was performed by the TaqMan miRNA assays (Applied Biosystems, USA) and U6 was used as an internal control. To measure circRNA expression, RNA was reverse transcribed to cDNA using the PrimeScript RT Reagent (Takara, Japan) and then quantified by real-time PCR using the SYBR Premix Ex Taq ${ }^{\text {TM }}$ (Takara, Japan). GAPDH was used as an internal control. The $2^{-\triangle A C t}$ method was applied to quantify gene expression. The primer sequences were as follows: circANKS1B: (forward) 5》GAAACCGTCACTGGAGAATTATCA-3囚, (reverse) 5囚-AAAGCTGCTTCATGAAGTGCAC-3®; GAPDH (forward) 5囚-GAACGGGAAGC- TCACTGG-3囚, (reverse) 5囚-GCCTGCTTCACCACCTTCT-3囚.

\section{Cell Migration and Invasion Assays}

For the migration assays, $5 \times 10^{4}$ cells in serum-free medium were placed in the upper chamber of the transwell (24-well insert, 8- $\mu \mathrm{m}$ pore size, BD Biosciences, San Jose, USA). For the invasion assays, cells with $200 \mathrm{~mL}$ of serum-free medium were placed in the upper chambers coated with Matrigel (BD Bioscience, San Jose, USA) according to the manufacturer's protocol. Media containing 20\% FBS were added to the lower chamber as a chemoattractant. After the cells had been incubated for 24 hours at $37^{\circ} \mathrm{C}$, the cells remaining on the upper membrane were removed and those on the lower surface of the membrane were fixed in $95 \%$ ethanol $\llbracket$ stained with $0.1 \%$ crystal violet photographed $(\times 200)$ in five independent fields for each well.

\section{Plasmid Construction and Dual-Luciferase Assay}

The wild type (wt) and mutation (mut) of circANKS1B-3邓UTR that contained the putative miR-152-3p binding sites were cloned into the pmirGLO (Promega, USA), as pmirGLO-wt-circANKS1B or pmirGLO-mutcircANKS1B. Cells were co-transfected with miRNA-152-3p mimics or NC and wt-circANKS1B or mutcircANKS1B, respectively. After 48 hours, the cells were lysed and assayed with a Dual Luciferase Reporter Assay Kit (Promega, USA) according to the manufacturer's instructions.

\section{Protein Isolation and Western Blot}

Total protein was collected by Total Protein Extraction Kit (KeyGen, China) and the concentration of protein was measured with a BCA Protein Detection Kit (Sigma, USA). The lysate was applied to a $12 \%$ polyacrylamide gel for electrophoresis experiments, and after electrophoresis, it was transferred to a PVDF membrane. Membranes were blocked using $5 \%$ milk and incubated with primary antibodies at $4^{\circ} \mathrm{C}$ overnight. After that, it was incubated with secondary antibody for $2-3 \mathrm{~h}$ at $37^{\circ} \mathrm{C}$. Signals were detected 
using enhanced chemiluminescence detection reagent (Thermo Scientific). Protein levels were determined by normalization to GAPDH.

\section{Statistical analysis}

Data are presented as the mean \pm standard deviation (SD) of at least three independent experiments. Student's $t$ test and one-way analysis of variance (ANOVA) were used to analyze significant differences. The Kaplan-Meier method was used to draw survival curves and the log-rank test was used to determine statistical significance. All of the statistical analyses were performed using SPSS 22.0 (SPSS Inc., USA). All $\mathrm{P}<0.05$ were marked with *, and $\mathrm{P}<0.01$ with **.

\section{Results}

\section{CircANKS1B was significantly overexpressed and indicated poor prognosis in PC}

To detect the expression of circANKS1B in PC, we firstly performed real-time qRT-PCR in cell lines and found that circANKS1B expression in PC cell lines (C4-2, LNCaP, DU145 and PC-3) was significantly higher than that in human prostate epithelial cell line (RWPE-1) (Fig. 1A). Then we analyzed circANKS1B expression in tissue samples. As the Fig. 1B showed, compared with matched adjacent non-tumor tissues, the expression of circANKS1B was remarkably up-regulated in PC tissues $(P<0.001)$. In addition, we performed Chi-square tests to analyze the association between the clinicopathological characteristics and circANKS1B expression. As shown in Table 1, circANKS1B expression was significantly associated with Gleason score $(P=0.019)$, T stage $(P=0.011)$ and Lymph node metastasis $(P=0.004)$. More importantly, high circANKS1B expression indicated poor overall survival $(P=0.001, \mathrm{Fig}$. 1C). These findings showed that circANKS1B could be a potential prognostic biomarker for PC patients. 
Table 1

Correlation between circANKS1B expression and clinicopathological characteristics in prostate cancer $(n=42)$

\begin{tabular}{|c|c|c|c|}
\hline Characteristics & CircANKS1B ex & ression & $P$-value \\
\hline & $\operatorname{Low}(21,50 \%)$ & High $(21,50 \%)$ & \\
\hline Age (years) & & & 0.739 \\
\hline$凶 65$ & $7(53.8 \%)$ & $6(46.2 \%)$ & \\
\hline$\geq 65$ & $14(48.3 \%)$ & $15(51.7 \%)$ & \\
\hline Gleason score & & & 0.019 \\
\hline ष7 & $10(76.9 \%)$ & $3(23.1 \%)$ & \\
\hline$\geq 7$ & $11(37.9 \%)$ & $18(62.1 \%)$ & \\
\hline T stage & & & 0.011 \\
\hline $\mathrm{T} 1-\mathrm{T} 2$ & $17(65.4 \%)$ & $9(34.6 \%)$ & \\
\hline T3-T4 & $4(25.0 \%)$ & $12(75.0 \%)$ & \\
\hline Lymph node metastasis & & & 0.004 \\
\hline No & $18(66.7 \%)$ & $9(33.3 \%)$ & \\
\hline Yes & $3(20.0 \%)$ & $12(80.0 \%)$ & \\
\hline
\end{tabular}

\section{Circanks1b Promoted Cell Migration And Invasion In Pc Cells}

Due to DU145 and PC3 cell lines showed a relatively higher expression of circANKS1B and were chose for the functional experiments. To investigate the role of circANKS1B in PC cells, we utilized circANKS1BsiRNA (si-circANKS1B) to down-regulate the expression of circANKS1B. As the Fig. 2A showed, both in DU145 and PC3 cells, the relative expression of circANKS1B dramatically decreased after transfecting with si-circANKS1B. Transwell assay demonstrated that silencing sircANKS1B effectively restrained the migration of DU145 and PC3 cells (Fig. 2B). Similarly, PC cells transfected with si-circANKS1B presented with a lower invasive capability than controls (Fig. 2C), suggesting that circANKS1B might play crucial roles in PC progression.

CircANKS1B functioned as the sponge of miR-152-3p in PC cells

To explore the mechanisms of sircANKS1B regulating PC progression, we performed a bioinformatic research (Starbase, CircNet database and Circlnteractome database) and found circANKS1B might have 
a putative binding site for miR-152-3p (Fig. 3A). As the Fig. 3B showed, after silencing sircANKS1B in DU145 and PC3 cells, the expression of miR-152-3p was significantly increased, indicating that circANKS1B negatively regulated miR-152-3p expression. To further identify the direct interaction between miR-152-3p and circANKS1B, we created the dual-luciferase reporters containing wild-type and mutative 3هUTR sequence of circANKS1B. DU145 and PC3 cells were co-transfected with miR-152-3p mimics or NC and pmirGLO-wt-circANKS1B-3区UTR (wt-circANKS1B) or pmirGLO-mut- circANKS1B-3囚UTR (mutcircANKS1B). The results showed that co-transfection of miR-152-3p mimics and wt-circANKS1B dramatically reduced the luciferase activity both in DU145 and PC3 cells. However, no significant changes were observed in PC cells co-transfecting with miR-152-3p mimics and mut-circANKS1B (Fig. 3C). The above data demonstrated that circANKS1B exerted its function by sponging miR-152-3p.

\section{Circanks1b Sponged And Sequestered Mir-152-3p To Up- regulate Tgf- $a$ Expression}

Our previous study has suggested that miR-152-3p suppressed PC cells migration and invasion by directly targeting TGF- $a$ and then down-regulated EGFR [14]. Thus, we performed the rescue experiments to explore whether circANKS1B regulated the migration and invasion of PC cells by the circANKS1B-miR152-3p-TGF-a pathway. Our results suggested that silencing circANKS1B significantly suppressed the expression of TGF- $a$ and EGFR, while the effects were reversed by knockdown of miR-152-3p expression through transfecting with miR-152-3p inhibitor in DU145 and PC3 cells (Fig. 4A). The traswell assay showed that circANKS1B deficiency dramatically inhibited cell invasion and the effects were abrogated by silencing miR-152-3p through transfecting with miR-152-3p inhibitor, while TGF-a deficiency reversed the impact of miR-152-3p inhibitor by transfecting with si-TGF-a in DU145 and PC3 cells (Fig. 4B). Our findings indicated that circANKS1B facilitated cell invasion of PC through up-regulating TGF-a and EGFR expression by competing for miR-152-3p.

\section{Discussion}

In 1976, circRNAs were discovered by electron microscopy of plant viroid for the first time [15]. Up to now, the advent of high-throughput sequencing associated with new computational pipelines to map circRNAs to the genome have moved circRNAs to the forefront of RNA research $[16,17]$. Emerging evidence showed that circRNAs exhibited aberrant expression and played critical roles in many types of cancer, including prostate cancer [18-21]. Recently, studies reported that circANKS1B was overexpressed and involved in tumorous progression in breast cancer [12] and colorectal cancer [13], respectively.

Our study firstly explored the expression and function of circANKS1B in PC. We found that the expression of circANKS1B was dramatically up-regulated both in PC cells and tissues. Moreover, high circANKS1B expression was associated with Gleason score, $T$ stage as well as lymph node metastasis and indicated poor prognosis of PC patients. Most of the circRNAs were found to be abundant, conserved and often tissue-specific [8]. Furthermore, circRNAs were greatly stable both inside cells as well as in extracellular 
plasma, including blood and saliva, and were also transported by exosomes from the cell body to extracellular fluid $[22,23]$. These properties of circRNAs showed great potential as biomarkers. Consequently, combining with our findings, circANKS1B would be a novel prognostic biomarker for PC patients.

In this study, we also observed that circANKS1B significantly promoted cell migration and invasion in PC cell lines. Furthermore, the expression of circANKS1B in PC tissues was also associated with poor overall survival of PC patients, suggesting circANKS1B participated in the progression of PC. However, what is the mechanism of circANKS1B involved in the regulation of progression in PC?

Growing evidence showed that circRNAs, as the sponge of miRNAs, participated in the initiation and progression of tumours through regulating expressions of miRNA-inhibited downstream genes [24]. For instance, in PC, circAMOTL1L, circABCC4 and circHIPK3 exerted their functions by sponging miR-193a-5p, miR-1182 and miR-338-3p, respectively $[18,19,24]$. In current study, bioinformatic analyses indicated that circANKS1B contained a putative binding site for miR-152-3p and silencing sircANKS1B could enforce the expression of miR-152-3p. More importantly, dual-luciferase reporter assay confirmed the direct interaction between miR-152-3p and circANKS1B. These findings indicated that circANKS1B also functioned as a sponge of miR-152-3p in PC cells.

Our previous study has suggested that miR-152-3p suppressed PC cells migration and invasion by downregulating TGF- $\alpha$ [14]. The aberrant expression of TGF- $a$ and TGF-a-induced signaling was involved in the progression and metastasis of various carcinomas [25-27]. TGF-a was found to bind to EGFR ligands and activate EGFR by inducing EGFR tyrosine autophosphorylation [28]. Activation of EGFR played critical roles in cell proliferation, survival, angiogenesis, migration, and invasion [29]. So we speculated that circANKS1B probably facilitated PC progression through regulating TGF- $a$ and EGFR expression by sequestering miR-152-3p. Supporting evidence from our study showed that silencing circANKS1B significantly suppressed the expression of TGF- $a$ and EGFR, while the effects were reversed by miR-152$3 p$ deficiency. Moreover, miR-152-3p deficiency also reversed the impacts of circANKS1B silencing on PC cell invasion, while this phenomenon was abrogated by TGF-a deficiency. Obviously, our findings confirmed our conjecture.

Taken together, this study firstly identified circANKS1B was significantly overexpressed and indicated poor prognosis in PC. Furthermore, circANKS1B as the sponge of miR-152-3p promoted PC cells migration and invasion by up-regulating TGF-a expression. Our findings reveal that circANKS1B could be a potential prognostic biomarker and therapeutic target of PC.

\section{Conclusions}

CircANKS1B as the sponge of miR-152-3p promots PC progression by up-regulating TGF-a expression and its high expression indicates poor prognosis of PC patients, suggesting circANKS1B may be a potential prognostic biomarker and therapeutic target for PC patients. 


\section{Abbreviations}

PC, prostate cancer; SD, standard deviation; ANOVA, one-way analysis of variance; si-RNA, small interfering-RNA; si-circANKS1B, si-RNA-circANKS1B; si-TGF-a, si-RNA-TGF-a; NC, negative control; wtcircANKS1B (Wt), wild type circANKS1B; mut-circANKS1B (Mut), mutative circANKS1B.

\section{Declarations}

\section{Authors' contributions}

LST and CZL designed this study and edited the manuscript. LJT and XYP researched literature, performed experiments and wrote the manuscript. JWW collected the tissue samples. LZ assembled and analyzed the data. All authors read and approved the final manuscript.

\section{Acknowledgments}

We would like to thank Chen Zhu from the Urology Department of Jiangsu Province Hospital of Chinese Medicine for his great help.

\section{Funding}

No funding was received.

\section{Availability of data and materials}

The data used to support the findings of this study were available from the corresponding author upon request.

\section{Ethics approval and consent to participate}

The study involving human participants were reviewed and approved by the Ethics Committee of the Second people囚hospital of Wuhu. The patients/participants provided their written informed consent to participate in this study. Written informed consent was obtained from the individual(s) for the publication of any potentially identifiable images or data included in this article.

\section{Consent for publication}

Not applicable.

\section{Competing interests}

None declared.

\section{Author details}


${ }^{1}$ Department of Urology, The First Affiliated Hospital of Anhui Medical University; Institute of Urology and Anhui Province Key Laboratory of Genitourinary Diseases, Anhui Medical University, Hefei 230022, Anhui, P.R. China; ${ }^{2}$ Department of Ophthalmology, The Second People's Hospital of Wuhu, Wuhu 241000, Anhui, P.R. China; ${ }^{3}$ Department of Urology, The Affiliated Wuhu No. 2 People's Hospital of Wannan Medical College, Wuhu 241000, Anhui, P.R. China.

\section{References}

1. Bray F, Ferlay J, Soerjomataram I, Siegel RL, Torre LA, Jemal A. Global cancer statistics 2018 : GLOBOCAN estimates of incidence and mortality worldwide for 36 cancers in 185 countries. CA Cancer J Clin. 2018;68(6):394-424.

2. Siegel RL, Miller KD, Jemal A. Cancer statistics. 2019. CA Cancer J Clin. 2019; 69(1): 7-34.

3. Pang C, Guan Y, Li H, Chen W, Zhu G. Urologic cancer in China. Jpn J Clin Oncol. 2016;46(6):497501.

4. Kristensen LS, Hansen TB, Venø MT, Kjems J. Circular RNAs in cancer: opportunities and challenges in the field. Oncogene. 2018;37(5):555-65.

5. Hsiao KY, Sun HS, Tsai SJ. Circular RNA - New member of noncoding RNA with novel functions. Exp Biol Med (Maywood). 2017;242(11):1136-41.

6. Li X, Yang L, Chen LL. The Biogenesis, Functions, and Challenges of Circular RNAs. Mol Cell. 2018;71(3):428-42.

7. Panda AC, Grammatikakis I, Munk R, Gorospe M, Abdelmohsen K. Emerging roles and context of circular RNAs. Wiley Interdiscip Rev RNA. 2017; 8(2).

8. Meng S, Zhou H, Feng Z, Xu Z, Tang Y, Li P, et al. CircRNA: functions and properties of a novel potential biomarker for cancer. Mol Cancer. 2017;16(1):94.

9. Haque S, Harries LW. Circular RNAs (circRNAs) in Health and Disease. Genes (Basel). 2017; 8(12).

10. Panda AC. Circular RNAs Act as miRNA Sponges. Adv Exp Med Biol. 2018;1087:67-79.

11. Rybak-Wolf, Stottmeister C, Glažar P, Jens M, Pino N, Giusti S, et al. Circular RNAs in the Mammalian Brain Are Highly Abundant, Conserved, and Dynamically Expressed. Mol Cell. 2015;58(5):870-85.

12. Zeng $K, \mathrm{He} B$, Yang BB, Xu T, Chen $X, X u M$, et al. The pro-metastasis effect of circANKS1B in breast cancer. Mol Cancer. 2018;17(1):160.

13. Li D, Yang R, Yang L, Wang D, Zhou X, Sun Y. circANKS1B regulates FOXM1 expression and promotes cell migration and invasion by functioning as a sponge of the miR-149 in colorectal cancer. Onco Targets Ther. 2019;12:4065-73.

14. Zhu C, Li J, Ding Q, Cheng G, Zhou H, Tao L, et al. miR-152 controls migration and invasive potential by targeting TGFa in prostate cancer cell lines. Prostate. 2013;73(10):1082-9.

15. Sanger HL, Klotz G, Riesner D, Gross HJ, Kleinschmidt AK. Viroids are single-stranded covalently closed circular RNA molecules existing as highly base-paired rod-like structures. Proc Natl Acad Sci U S A. 1976;73(11):3852-6. 
16. Panda AC, De S, Grammatikakis I, Munk R, Yang X, Piao Y, et al. High-purity circular RNA isolation method (RPAD) reveals vast collection of intronic circRNAs. Nucleic Acids Res. 2017;45(12):e116.

17. Memczak S, Jens M, Elefsinioti A, Torti F, Krueger J, Rybak A, et al. Circular RNAs are a large class of animal RNAs with regulatory potency. Nature. 2013;495(7441):333-8.

18. Yang Z, Qu CB, Zhang Y, Zhang WF, Wang DD, Gao CC, et al. Dysregulation of p53-RBM25-mediated circAMOTL1L biogenesis contributes to prostate cancer progression through the circAMOTL1L-miR193a-5p-Pcdha pathway. Oncogene. 2019;38(14):2516-32.

19. Huang $C$, Deng $H$, Wang $Y$, Jiang $H, X u R$, Zhu X, et al. Circular RNA circABCC4 as the ceRNA of miR1182 facilitates prostate cancer progression by promoting FOXP4 expression. J Cell Mol Med. 2019;23(9):6112-9.

20. Shen Z, Zhou L, Zhang C, Xu J. Reduction of circular RNA Foxo3 promotes prostate cancer progression and chemoresistance to docetaxel. Cancer Lett. 2020;468:88-101.

21. Feng $Y$, Yang Y, Zhao X, Fan Y, Zhou L, Rong J, et al. Circular RNA circ0005276 promotes the proliferation and migration of prostate cancer cells by interacting with FUS to transcriptionally activate XIAP. Cell Death Dis. 2019;10(11):792.

22. Bahn JH, Zhang Q, Li F, Chan TM, Lin X, Kim Y, et al. The landscape of microRNA, Piwi-interacting RNA, and circular RNA in human saliva. Clin Chem. 2015;61(1):221-30.

23. Li Y, Zheng Q, Bao C, Li S, Guo W, Zhao J, et al. Circular RNA is enriched and stable in exosomes: a promising biomarker for cancer diagnosis. Cell Res. 2015;25(8):981-4.

24. Cai C, Zhi Y, Wang K, Zhang P, Ji Z, Xie C, et al. CircHIPK3 overexpression accelerates the proliferation and invasion of prostate cancer cells through regulating miRNA-338-3p. Onco Targets Ther. 2019;12:3363-72.

25. Lau TS, Chan LK, Wong EC, Hui CW, Sneddon K, Cheung TH, et al. A loop of cancer-stroma-cancer interaction promotes peritoneal metastasis of ovarian cancer via TNFa-TGFa-EGFR. Oncogene. 2017;36(25):3576-87.

26. Dopeso H, Jiao HK, Cuesta AM, Henze AT, Jurida L, Kracht M, et al. PHD3 Controls Lung Cancer Metastasis and Resistance to EGFR Inhibitors through TGFa. Cancer Res. 2018;78(7):1805-19.

27. Sun J, Cui H, Gao Y, Pan Y, Zhou K, Huang J, et al. TGF-a Overexpression in Breast Cancer Bone Metastasis and Primary Lesions and TGF-a Enhancement of Expression of Procancer Metastasis Cytokines in Bone Marrow Mesenchymal Stem Cells. Biomed Res Int. 2018; 2018: 6565393.

28. Salomon DS, Brandt R, Ciardiello F, Normanno N. Epidermal growth factor-related peptides and their receptors in human malignancies. Crit Rev Oncol Hematol. 1995;19(3):183-232.

29. Yarden Y, Sliwkowski MX. Untangling the ErbB signalling network. Nat Rev Mol Cell Biol. 2001;2(2):127-37.

\section{Figures}


A

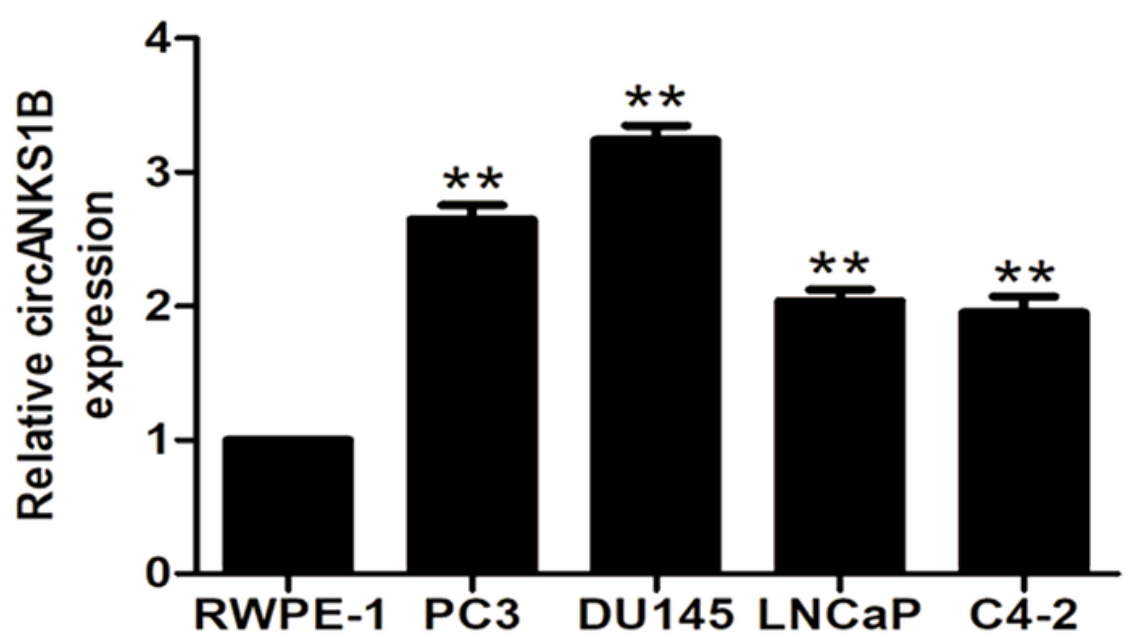

B

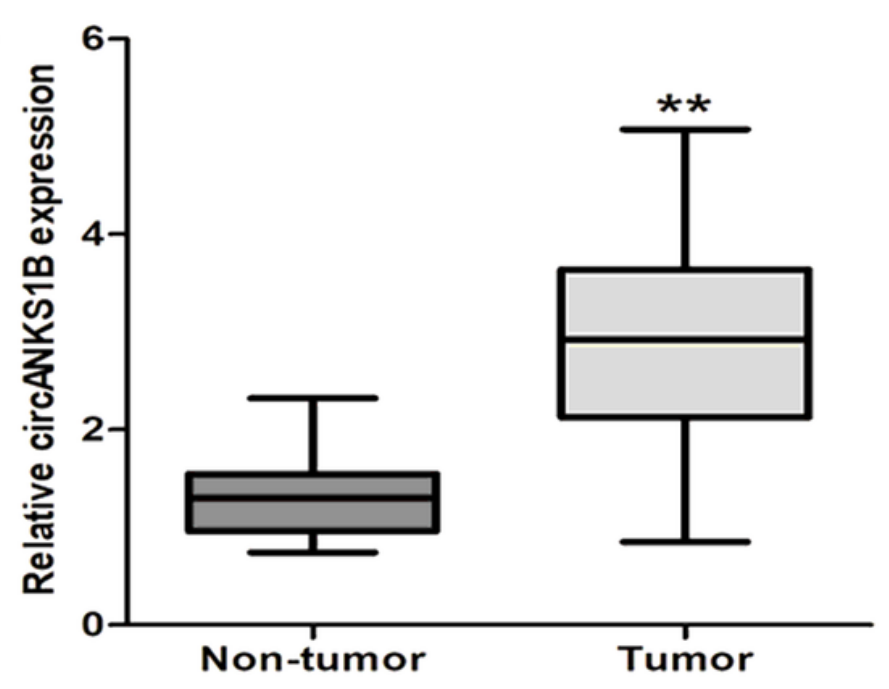

C

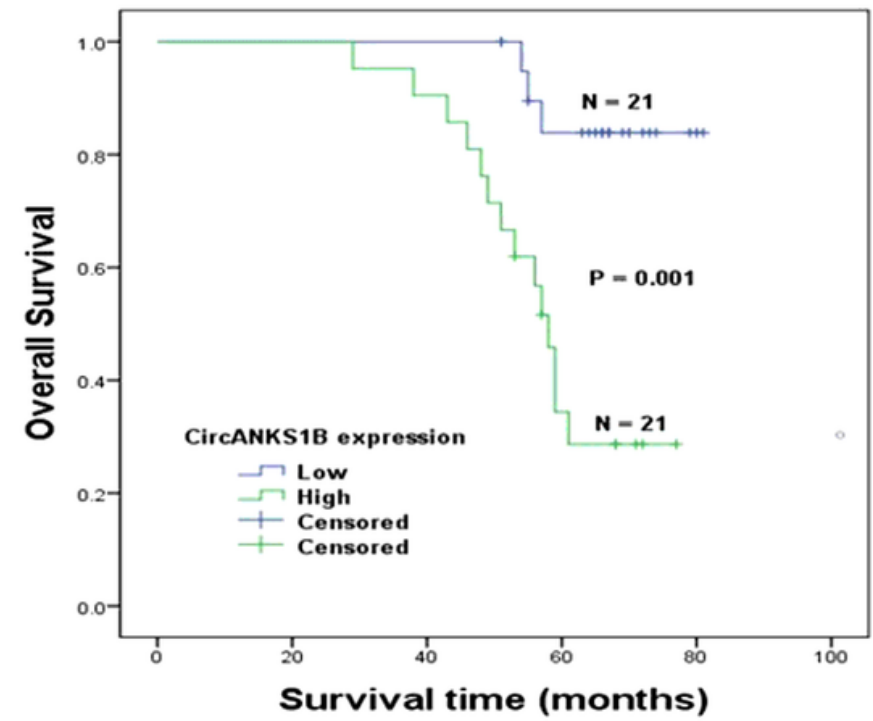

Figure 1

CircABKS1B was overexpressed in PC cells and tissues. (A) Relative expression of circANKS1B in human PC cell lines (C4-2, LNCaP, DU145 and PC-3) and human prostate epithelial cell line (RWPE-1) detected by real time qRT-PCR. (B) Relative expression of circANKS1B in 42 pairs PC tissues and matched non-tumor tissues detected by real time qRT-PCR. (C) Kaplan-Meier survival analysis based on the expression of circANKS1B in PC tissues. Data are represented as mean \pm SD of three independent experiments. ${ }^{*} \mathrm{P}<$ 0.01 . 
A

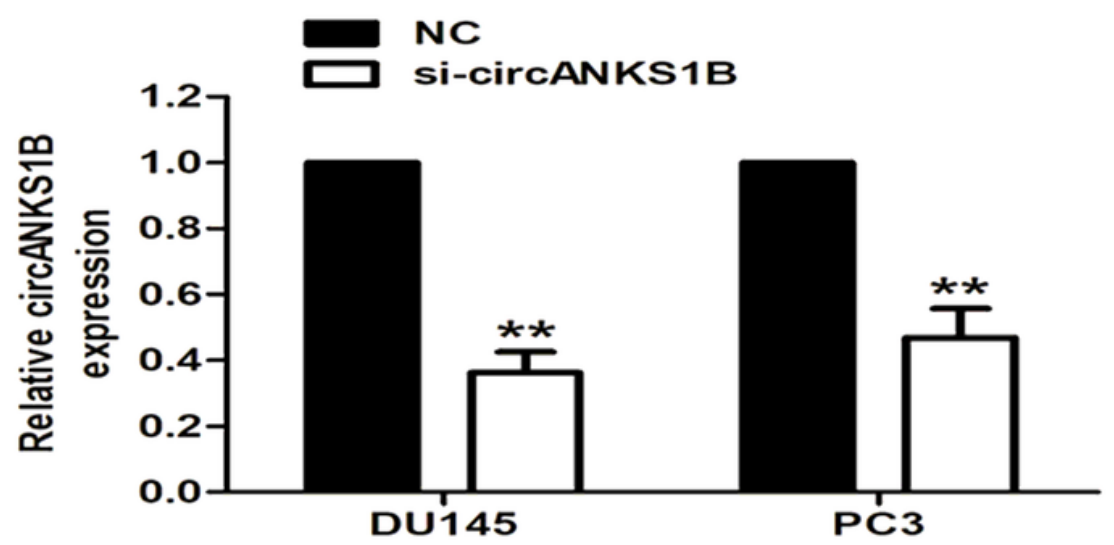

B

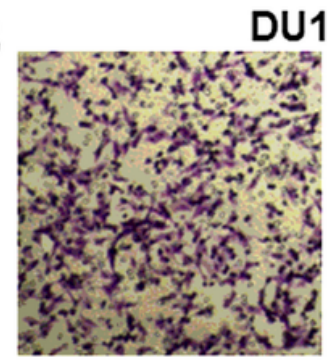

NC
DU145

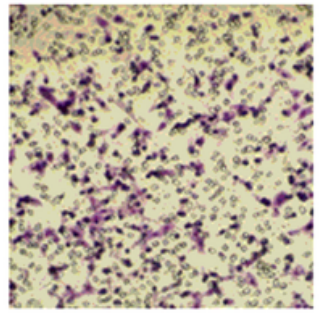

si-circANKS1B

C

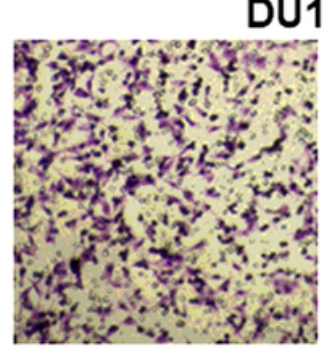

NC
DU145

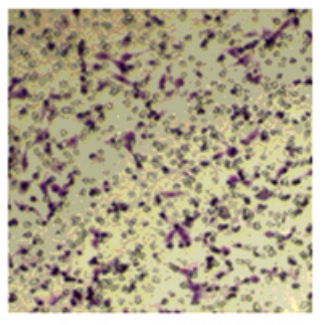

si-circANKS1B

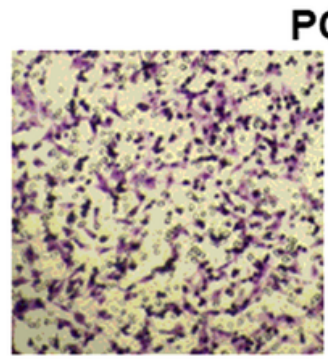

NC
PC3

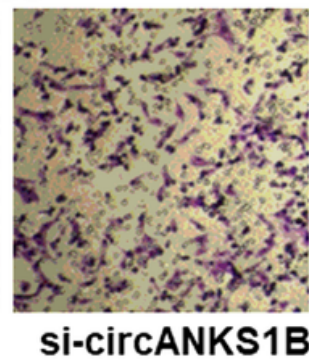

PC3

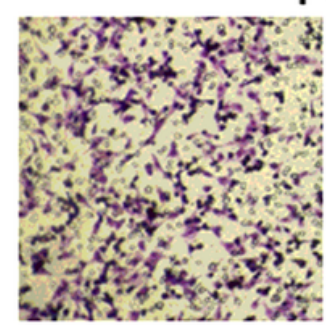

NC

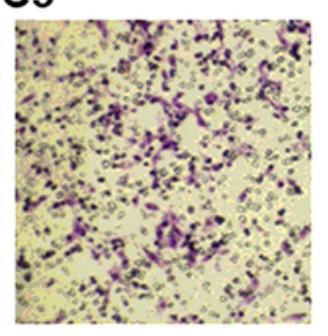

si-circANKS1B
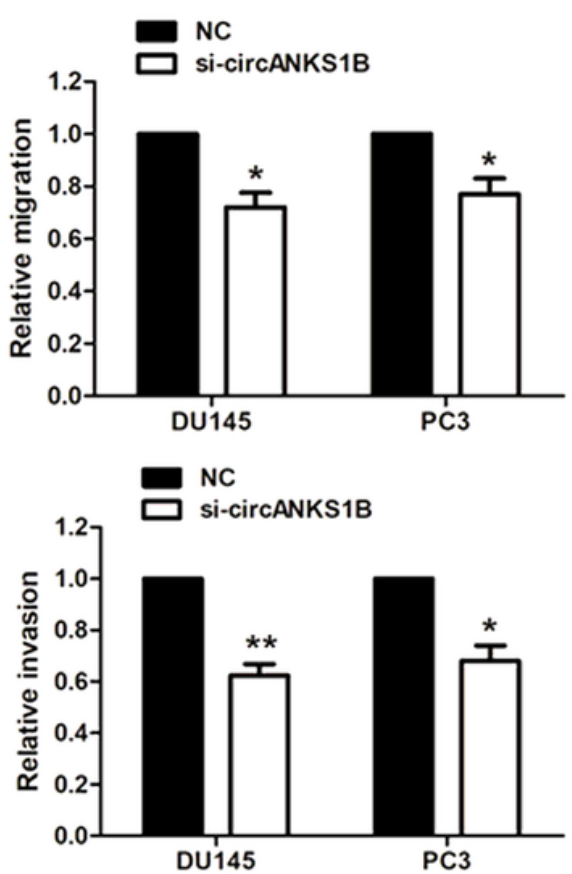

Figure 2

Silencing circANKS1B suppressed cell migration and invasion in PC cells. (A) Relative expression of circANKS1B was measured by real time qRT-PCR in DU145 and PC3 cells transfected with si-circANKS1B or NC. (B and C) The ability of cell migration (B) and invasion (C) were measured by transwell assay in DU145 and PC3 cells transfected with si-circANKS1B or NC. Data are represented as mean \pm SD of three independent experiments. ${ }^{*} \mathrm{P}<0.05 .{ }^{*} \mathrm{P}<0.01$. 
A wt-circANKS1B 5' -GGGGAGTGCACTT-3'

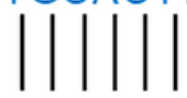

miR-152-3p 3' -GACAGUACGUGAC-5'

mut-circANKS1B 5' -GGGGAGCCATGGA-3'
B

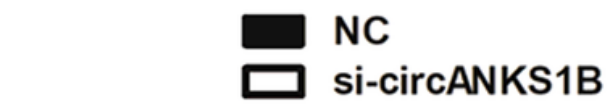

C NC

口 miR-152-3p

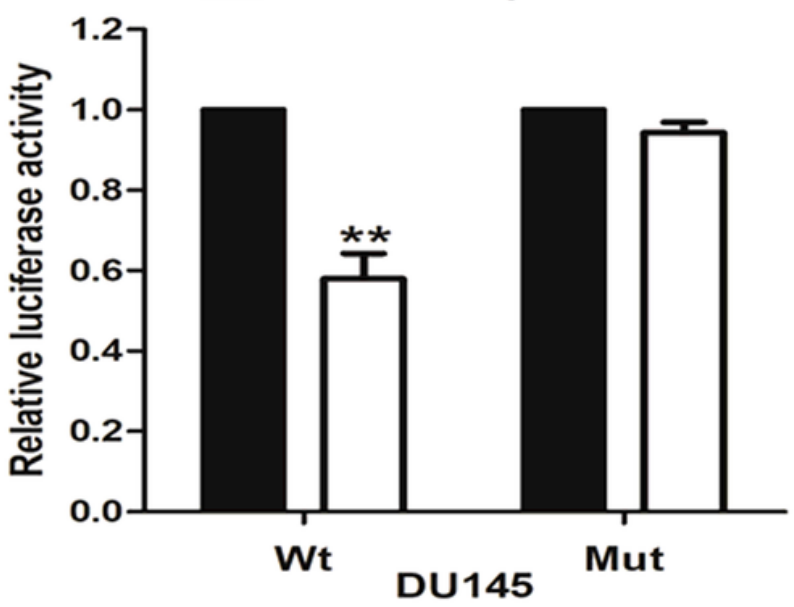

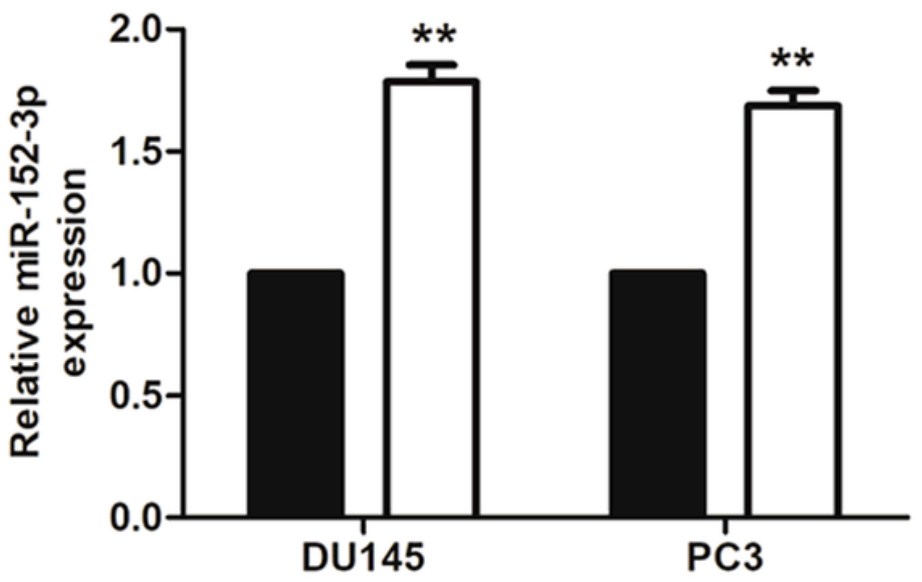

NC

$\square$ miR-152-3p

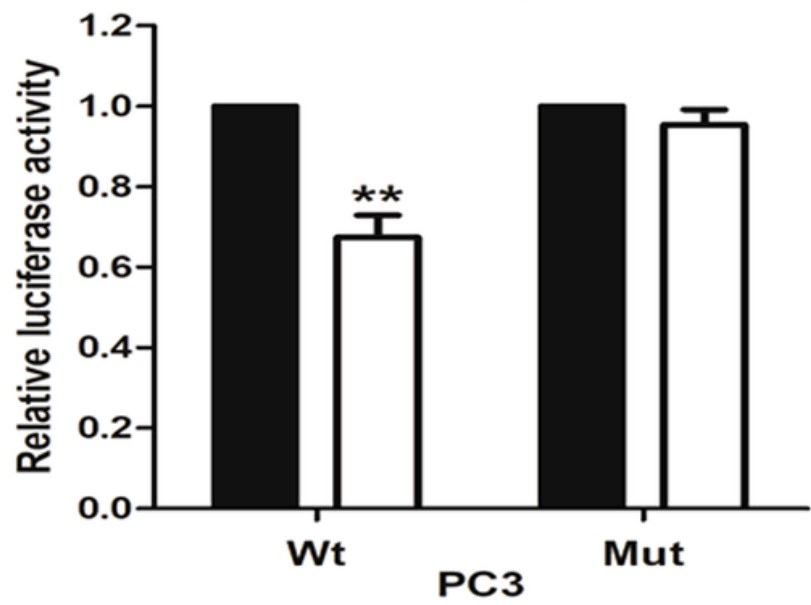

Figure 3

CircANKS1B functioned as a sponge for the miR-152-3p. (A) Predicted binding site of miR-152-3p in circANKS1B. (B) Relative expression of miR-152-3p was determined by real time qRT-PCR in DU145 and PC3 cells transfected with si-circANKS1B or NC. (C) Relative luciferase activity was determined by dualluciferase reporter assay in DU145 and PC3 cells co-transfected with miR-152-3p mimics or NC and wtcircANKS1B (Wt) or mut-circANKS1B (Mut). Data are represented as mean \pm SD of three independent experiments. ${ }^{\star *} \mathrm{P}<0.01$. 
A

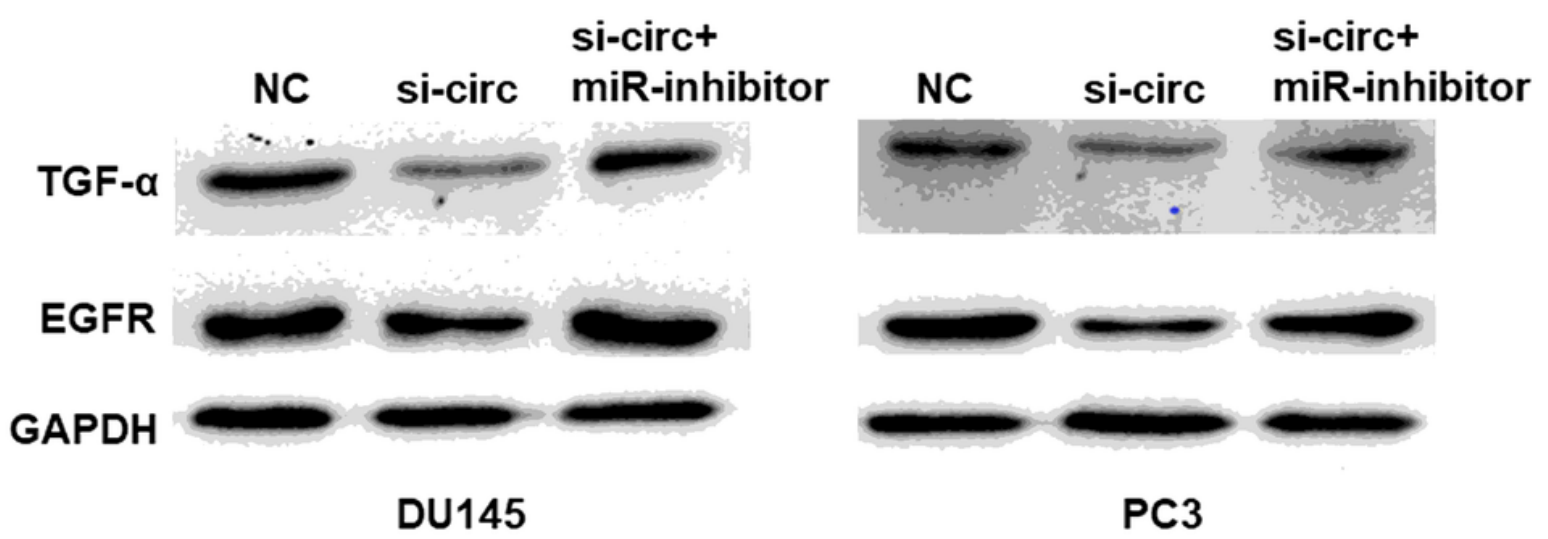

B

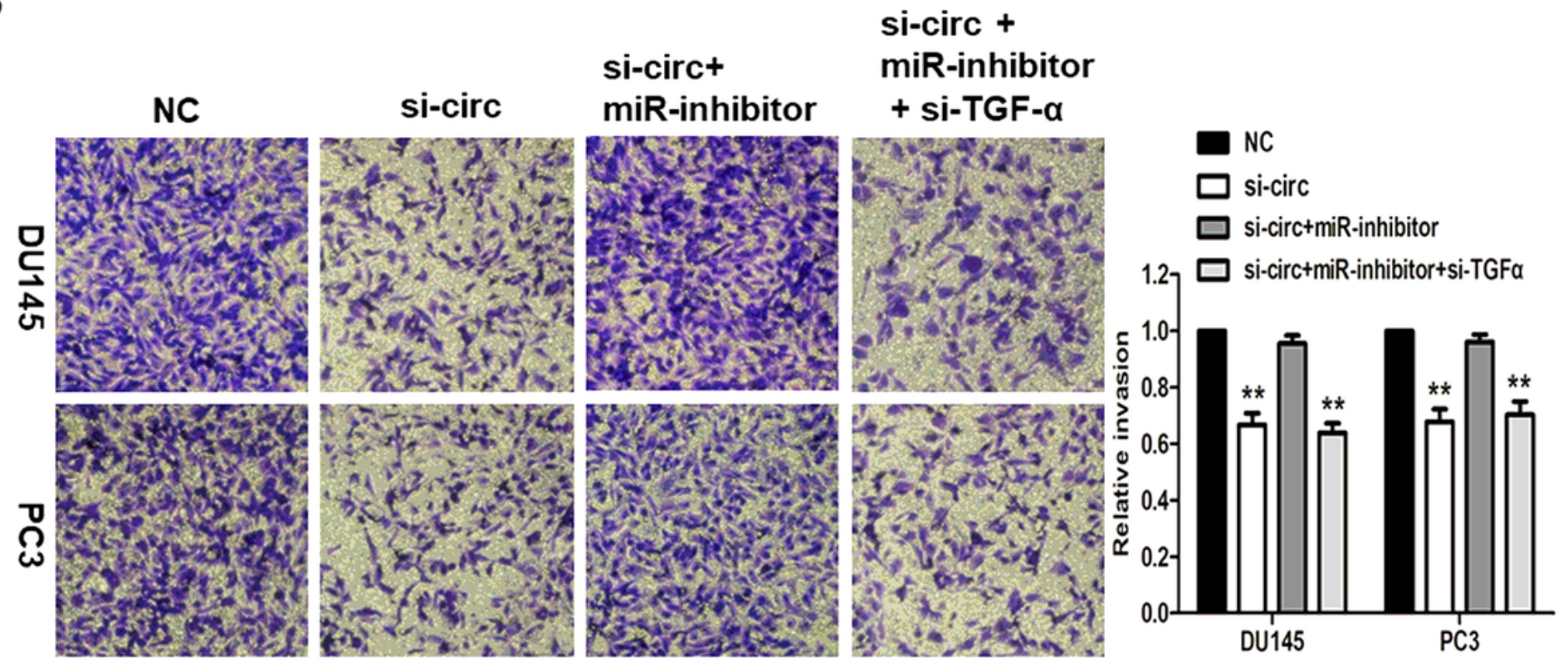

Figure 4

CircANKS1B promoted PC progression by modulating miR-152-2p-TGF-a pathway. (A) After corresponding transfection, expression of TGF- $a$ and EGFR were determined by western blotting assay in DU145 and PC3 cells. (B) After corresponding transfection, the ability of cell invasion was measured by transwell assay in DU145 and PC3 cells. Data are represented as mean \pm SD of three independent experiments. ${ }^{*} \mathrm{P}<0.01$. 